\title{
Scattering of a Plane Wave by an Anisotropic Plasma-Coated Conducting Sphere
}

\author{
You-Lin Geng \\ Institute of Antenna and Microwaves, Hangzhou Dianzi University, Xiasha, Zhejiang Province, Hangzhou 310018, China \\ Correspondence should be addressed to You-Lin Geng, gengyoulin@yahoo.com.cn
}

Received 19 June 2011; Revised 18 August 2011; Accepted 24 August 2011

Academic Editor: Miguel Ferrando

Copyright () 2011 You-Lin Geng. This is an open access article distributed under the Creative Commons Attribution License, which permits unrestricted use, distribution, and reproduction in any medium, provided the original work is properly cited.

The electromagnetic field in homogeneous plasma anisotropic medium can be expressed as the addition of the first and second spherical vector wave functions in plasma anisotropic medium. The tangential electromagnetic fields are continued in the boundary between the homogeneous plasma anisotropic medium and free space, and the tangential electrical field is zero in the surface of conducting sphere. The coefficients of electromagnetic fields in plasma anisotropic medium expanded in terms of spherical vector wave functions in plasma anisotropic medium are derived, and then the coefficients of scattering fields in terms of spherical vector functions in free space can be obtained. Numerical results between this paper and hybrid finite element-boundary integral-multilevel fast multipole algorithm (FE-BI-MLFMA) are given, and they are in agreement very well. Some new numerical results of a plane wave scattering by an anisotropic plasma-coated conducting sphere are obtained.

\section{Introduction}

Plasma represents a medium of gas in highly ionized state, and it is a neutral mixture of free ions, electrons, and molecules. For example, when the ionosphere and the sheath of satellite and missile come into atmosphere, they are considered to be plasma in nature. When there is an externally applied magnetic field, a plasma exhibits anisotropic behavior and its permittivity takes a tensor form. The elements of the permittivity tensor are complex functions of wave, plasma, gyro and collision frequencies [1-3]. Wave propagation in, and scattering by, an anisotropic medium is nonreciprocal, and its analysis becomes very difficult even when the plasma is treated as an incompressible gas [1-4].

The interaction between electromagnetic wave and anisotropic medium has attracted much interest. It is simply because there are many natural and artificial anisotropic materials, and they are frequently used in optical signal processing (such as constructing signal processing elements at optical frequencies), the radar cross-section control for various objects or scatterers, antennas or airborne radomes, optical fibers, developments of certain types of radar absorbers, and high-performance microstrip antenna designs where the substrates of this nature are desirable.
One of the basic problems in investigating waves in anisotropic media is to accurately and efficiently characterize electromagnetic scattering. Scattering by homogeneous anisotropic objects has attracted considerable interests in recent years. Numerical methods based on integral equations [5] as well as differential equations [6] were developed, and the analytical method based on eigen vector wave functions [7] was also adopted to characterize this kind of problems. Although the efforts in the past were primarily spent on twodimensional (2-D) geometries, some progresses have been made in the analysis of three-dimensional (3-D) anisotropic scatterers using the method of moments (MoM) $[8,9]$, the couple dipole approximation method [10], hybrid finite element-boundary integral-multilevel fast multipole algorithm (FE-BI-MLFMA) [11], integral equation method [12], and Fourier transform, eigenvalue and eigen functions, spherical vector wave functions solution [13-15]. In the present study, the simple and efficient method proposed in [13] will be employed to tackle the scattering problem associated with an anisotropic plasma-coated conducting sphere by a plane wave.

Electromagnetic fields in plasma anisotropic medium and free space can be expanded in terms of spherical vector wave functions in plasma anisotropic medium and free space, 


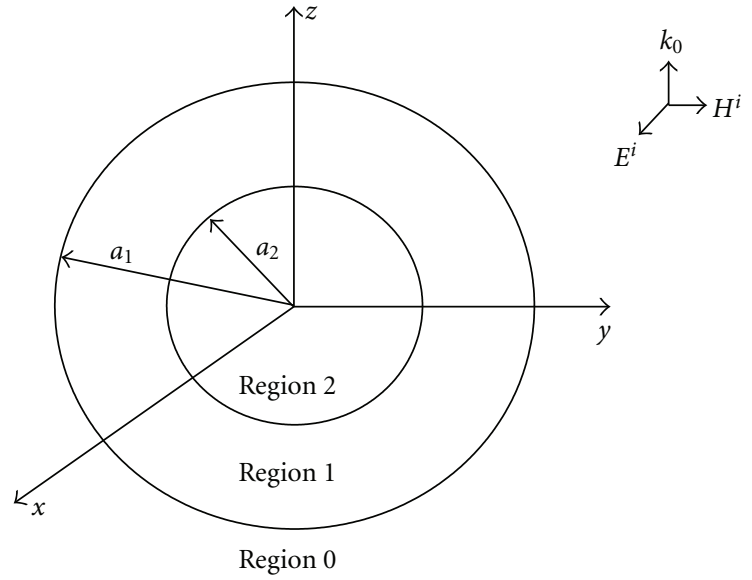

Figure 1: The geometry of scattering of a plane wave by an anisotropic plasma-coated conducting sphere.

respectively. Applying the continued boundary conditions of electromagnetic fields on the interfaces between the free space and a coated-plasma anisotropic medium, and the vanishing of the tangential field in the surface of the conducting sphere, the coefficients of electromagnetic fields in plasma anisotropic medium and scattered fields in free space can be obtained. Then, radar cross-sections of an anisotropic plasma-coated conducting sphere scattered by a plane wave can be derived. The theoretical analysis shows that the present method can be reduced to these of the homogeneous plasma anisotropic medium when the radius of conducting sphere approaches zero. This analytical solution to electromagnetic scattering by an anisotropic plasma-coated conducting sphere can be used to characterize the targets and their radar cross-sections and also to understand wireless communication channels and radio wave propagation mechanisms.

\section{Formulation of the Problem}

Consider the geometry depicted in Figure 1 which shows a cross-section of an anisotropic plasma-coated conducting sphere where the outer and inner radii of the plasma anisotropic medium are $a_{1}$ and $a_{2}$, respectively. Three distinct regions are thus divided into, namely, region 0 for the free space, region 1 for the plasma anisotropic spherical shell, and region 2 for the conducting sphere. This composite structure is illuminated by a plane electromagnetic wave (which is assumed to have an electric field amplitude equal to unity, to be polarized in parallel to the $x$-direction, and to propagate in the $z$-direction). In the following analysis, a time dependence of $\exp (-i \omega t)$ is assumed for the electromagnetic field quantities, but is suppressed throughout the treatment.

The electric field vector wave equation in such a sourcefree plasma anisotropic medium can be written in the following form $[7,12-15]$ :

$$
\nabla \times \nabla \times \mathbf{E}-\omega^{2} \mu_{0} \overline{\boldsymbol{\epsilon}} \cdot \mathbf{E}=0
$$

where $\mathbf{E}$ denotes the electric field, while $\mu_{0}$ represents the free-space permeability, and $\overline{\boldsymbol{\epsilon}}$ identifies the permittivity tensor of the plasma anisotropic medium. If an external constant magnetic filed is applied in the $+z$-direction, the permittivity tensor $\overline{\boldsymbol{\epsilon}}$ takes the form $[1-4,13,14]$ of

$$
\bar{\epsilon}=\left[\begin{array}{ccc}
\epsilon_{1} & -\epsilon_{2} & 0 \\
\epsilon_{2} & \epsilon_{1} & 0 \\
0 & 0 & \epsilon_{3}
\end{array}\right] .
$$

Using the Fourier transform as in [7, 12-15], the expansion of plane wave factors in terms of spherical vector wave functions of the first kind in isotropic medium [16] and the other three (the second, third, and fourth) kinds of spherical Bessel functions satisfying the same differential equations and the recursive relations of the first spherical Bessel functions, the electromagnetic fields (designated by the subscript 1 ) in the homogeneous plasma anisotropic spherical medium (region 1) can be obtained as follows:

$$
\begin{aligned}
& \mathbf{E}_{1}=\sum_{l=1}^{2} \sum_{q=1}^{2} \sum_{m n n^{\prime}} F_{m n^{\prime} q}^{(l)} \int_{0}^{\pi}[ A_{m n q}^{e}\left(\theta_{k}\right) \mathbf{M}_{m n}^{(l)}\left(\mathbf{r}, k_{q}\right) \\
&+B_{m n q}^{e}\left(\theta_{k}\right) \mathbf{N}_{m n}^{(l)}\left(\mathbf{r}, k_{q}\right) \\
&\left.+C_{m n q}^{e}\left(\theta_{k}\right) \mathbf{L}_{m n}^{(l)}\left(\mathbf{r}, k_{q}\right)\right] \\
& \times P_{n^{\prime}}^{m}\left(\cos \theta_{k}\right) k_{q}^{2} \sin \theta_{k} d \theta_{k}, \\
& \mathbf{H}_{1}=\sum_{l=1}^{2} \sum_{q=1}^{2} \sum_{m n n^{\prime}} F_{m n^{\prime} q}^{(l)} \int_{0}^{\pi}\left[A_{m n q}^{h}\left(\theta_{k}\right) \mathbf{M}_{m n}^{(l)}\left(\mathbf{r}, k_{q}\right)\right. \\
&+B_{m n q}^{h}\left(\theta_{k}\right) \mathbf{N}_{m n}^{(l)}\left(\mathbf{r}, k_{q}\right) \\
&\left.+C_{m n q}^{h}\left(\theta_{k}\right) \mathbf{L}_{m n}^{(l)}\left(\mathbf{r}, k_{q}\right)\right] \\
& \times P_{n^{\prime}}^{m}\left(\cos \theta_{k}\right) k_{q}^{2} \sin \theta_{k} d \theta_{k},
\end{aligned}
$$

where $n^{\prime}$ and $n$ are summed up both from 0 to $+\infty$ while $m$ is summed up from $-n$ to $n$; $\mathbf{r}$ denotes a position vector in the spherical coordinates; the coefficients, $F_{m n^{\prime} q}^{(l)}$, are unknown to be determined using the boundary conditions as in $[13,14]$; finally expansion coefficient $A_{m n q}^{p}\left(\theta_{k}\right), B_{m n q}^{p}\left(\theta_{k}\right), C_{m n q}^{p}\left(\theta_{k}\right)$ (where $p=e$ or $h$ ) and the eigenvalues of the coated plasma anisotropic medium $k_{q}(q=1,2)$ are all functions of $\theta_{k}$ and they have been derived in [13]. The vectors, $\mathbf{M}_{m n}^{(l)}, \mathbf{N}_{m n}^{(l)}$, and $\mathbf{L}_{m n}^{(l)}$, denote spherical vector wave functions in isotropic medium and they are defined as follows [13-16]:

$$
\begin{aligned}
\mathbf{M}_{m n}^{(l)}= & z_{n}^{(l)}(k r)\left[i m \frac{P_{n}^{m}(\cos \theta)}{\sin \theta} \hat{\boldsymbol{\theta}}-\frac{d P_{n}^{m}(\cos \theta)}{d \theta} \hat{\boldsymbol{\phi}}\right] e^{i m \phi}, \\
\mathbf{N}_{m n}^{(l)}= & n(n+1) \frac{z_{n}^{(l)}(k r)}{k r} P_{n}^{m}(\cos \theta) e^{i m \phi} \widehat{\mathbf{r}} \\
& +\frac{1}{k r} \frac{d\left(r z_{n}^{(l)}(k r)\right)}{d r} \cdot\left[\frac{d P_{n}^{m}(\cos \theta)}{d \theta} \hat{\boldsymbol{\theta}}+i m \frac{P_{n}^{m}(\cos \theta)}{\sin \theta} \hat{\boldsymbol{\phi}}\right] e^{i m \phi},
\end{aligned}
$$




$$
\begin{aligned}
\mathbf{L}_{m n}^{(l)}=k\{ & \frac{d z_{n}^{(l)}(k r)}{d(k r)} P_{n}^{m}(\cos \theta) \hat{\mathbf{r}}+\frac{z_{n}^{(l)}(k r)}{k r} \\
& \left.\times\left[\frac{d P_{n}^{m}(\cos \theta)}{d \theta} \hat{\boldsymbol{\theta}}+i m \frac{P_{n}^{m}(\cos \theta)}{\sin \theta} \hat{\boldsymbol{\phi}}\right]\right\} e^{i m \phi},
\end{aligned}
$$

where $z_{n}^{(l)}(x)$ (with $l=1,2,3$, and 4 ) denotes an appropriate kind of spherical Bessel functions, $j_{n}(x), y_{n}(x), h_{n}^{(1)}(x)$, and $h_{n}^{(2)}(x)$, respectively.

Equation (3) are the spherical vector wave functions in plasma anisotropic medium, which is different to that in isotropic medium which are shown in (4a) and (4b),

(i) first, spherical vector wave functions in plasma anisotropic medium is an integral expression comparing to that in isotropic medium, and

(ii) second, the eigenvalues $\left(k_{q}\right)$ in plasma anisotropic medium have two different values, but the wave number in isotropic medium is one $(k)$.

The spherical vector wave functions in plasma anisotropic medium is more complex than that in isotropic medium, and electromagetic fields in plasma anisotropic medium can be expressed by that in anisotropic medium.

To characterize the scattering properties of the plasmacoated conducting sphere, the incidence wave (designated by the superscript inc) and the scattered wave (designated by the superscript $s$ ) can be expressed in free space in terms of spherical vector wave functions as follows [13-16]:

$$
\begin{aligned}
& \mathbf{E}^{i n c}= \sum_{m n}\left[\delta_{m, 1}+\delta_{m,-1}\right] \\
& \times\left[a_{m n}^{x} \mathbf{M}_{m n}^{(1)}\left(\mathbf{r}, k_{0}\right)+b_{m n}^{x} \mathbf{N}_{m n}^{(1)}\left(\mathbf{r}, k_{0}\right)\right], \\
& \mathbf{H}^{i n c}= \frac{k_{0}}{i \omega \mu_{0}} \sum_{m n}\left[\delta_{m, 1}+\delta_{m,-1}\right] \\
& \times\left[a_{m n}^{x} \mathbf{N}_{m n}^{(1)}\left(\mathbf{r}, k_{0}\right)+b_{m n}^{x} \mathbf{M}_{m n}^{(1)}\left(\mathbf{r}, k_{0}\right)\right], \\
& \mathbf{E}^{s}=\sum_{m n}\left[A_{m n}^{s} \mathbf{M}_{m n}^{(3)}\left(\mathbf{r}, k_{0}\right)+B_{m n}^{s} \mathbf{N}_{m n}^{(3)}\left(\mathbf{r}, k_{0}\right)\right], \\
& \mathbf{H}^{s}=\frac{k_{0}}{i \omega \mu_{0}} \sum_{m n}\left[A_{m n}^{s} \mathbf{N}_{m n}^{(3)}\left(\mathbf{r}, k_{0}\right)+B_{m n}^{s} \mathbf{M}_{m n}^{(3)}\left(\mathbf{r}, k_{0}\right)\right],
\end{aligned}
$$

where $k_{0}$ denotes the wave number in free space, and the expansion coefficients of incident wave $a_{m n}^{x}, b_{m n}^{x}$, and $\delta_{m n}$ have been obtained earlier in [13-15] and are defined by

$$
\begin{gathered}
a_{m n}^{x}= \begin{cases}i^{n+1} \frac{2 n+1}{2 n(n+1)}, & m=1, \\
i^{n+1} \frac{2 n+1}{2}, & m=-1,\end{cases} \\
b_{m n}^{x}= \begin{cases}i^{n+1} \frac{2 n+1}{2 n(n+1)}, & m=1, \\
-i^{n+1} \frac{2 n+1}{2}, & m=-1,\end{cases} \\
\delta_{s, l}= \begin{cases}1, & s=l, \\
0, & s \neq l .\end{cases}
\end{gathered}
$$

The expansion coefficients of scattered fields, $A_{m n}^{s}$ and $B_{m n}^{s}$ in (6a) and (6b) ( $n$ varies from 0 to $+\infty$ while $m$ changes from $-n$ to $n$ ), are unknown to be determined using the boundary conditions, together with the unknown coefficients $F_{m n^{\prime} q}^{(l)}($ where $l=1,2$ and $q=1,2)$ in (3).

From (3), it shows that when the radius $a_{2}$ of the conductor sphere is infinitely small, the electromagnetic fields in the coating plasma anisotropic spherical shell origin are still finite, but the value of the second spherical Bessel functions becomes infinitely large in the origin; therefore the expansion coefficients $F_{m n^{\prime} q}^{(2)}$ of spherical vector wave functions in (3) will vanish, and in this connection, the present method in this paper can be automatically reduced to a homogeneous plasma anisotropic sphere, which is the same as that in [13].

By applying continuous boundary conditions of tangential electromagnetic field components on the interface between the plasma anisotropic medium and free space (where $r=a_{1}$ ) and utilizing the orthogonality of the tangential spherical vector wave functions [13], the following expressions are obtained:

$$
\begin{gathered}
\sum_{l=1}^{2} \sum_{q=1}^{2} \sum_{n^{\prime}=0}^{\infty} F_{m n^{\prime} q}^{(l)} \int_{0}^{\pi} A_{m n q}^{e} z_{n}^{(l)}\left(k_{q} a_{1}\right) P_{n^{\prime}}^{m}\left(\cos \theta_{k}\right) k_{q}^{2} \sin \theta_{k} d \theta_{k} \\
=\left[\delta_{m, 1}+\delta_{m,-1}\right] a_{m n}^{x} j_{n}\left(k_{0} a_{1}\right)+A_{m n}^{s} h_{n}^{(1)}\left(k_{0} a_{1}\right), \\
\sum_{l=1}^{2} \sum_{q=1}^{2} \sum_{n^{\prime}=0}^{\infty} F_{m n^{\prime} q}^{(l)} \int_{0}^{\pi}\left[B_{m n q}^{e} R_{n}^{(l)}\left(k_{q} r\right)+C_{m n q}^{e} \frac{z_{n}^{(l)}\left(k_{q} r\right)}{r}\right]_{r=a_{1}} \\
\quad \times P_{n^{\prime}}^{m}\left(\cos \theta_{k}\right) k_{q}^{2} \sin \theta_{k} d \theta_{k} \\
=\left[\delta_{m, 1}+\delta_{m,-1}\right] b_{m n}^{x} R_{n}^{(1)}\left(k_{0} a_{1}\right)+B_{m n}^{s} R_{n}^{(3)}\left(k_{0} a_{1}\right),
\end{gathered}
$$

$$
\begin{array}{r}
\sum_{l=1}^{2} \sum_{q=1}^{2} \sum_{n^{\prime}=0}^{\infty} F_{m n^{\prime} q}^{(l)} \int_{0}^{\pi} A_{m n q}^{h} z_{n}^{(l)}\left(k_{q} a_{1}\right) P_{n^{\prime}}^{m}\left(\cos \theta_{k}\right) k_{q}^{2} \sin \theta_{k} d \theta_{k} \\
=\frac{k_{0}}{i \omega \mu_{0}}\left\{\left[\delta_{m, 1}+\delta_{m,-1}\right] b_{m n}^{x} j_{n}\left(k_{0} a_{1}\right)+B_{m n}^{s} h_{n}^{(1)}\left(k_{0} a_{1}\right)\right\},
\end{array}
$$

$$
\begin{aligned}
\sum_{l=1}^{2} \sum_{q=1}^{2} \sum_{n^{\prime}=0}^{\infty} F_{m n^{\prime} q}^{(l)} \int_{0}^{\pi}\left[B_{m n q}^{h} R_{n}^{(l)}\left(k_{q} r\right)+C_{m n q}^{h} \frac{z_{n}^{(l)}\left(k_{q} r\right)}{r}\right]_{r=a_{1}} \\
\times P_{n^{\prime}}^{m}\left(\cos \theta_{k}\right) k_{q}^{2} \sin \theta_{k} d \theta_{k} \\
=\frac{k_{0}}{i \omega \mu_{0}}\left\{\left[\delta_{m, 1}+\delta_{m,-1}\right] a_{m n}^{x} R_{n}^{(1)}\left(k_{0} a_{1}\right)+A_{m n}^{s} R_{n}^{(3)}\left(k_{0} a_{1}\right)\right\} .
\end{aligned}
$$

Similarly on the interface of the conducting sphere (where $r=a_{2}$ ), the boundary condition requires the vanishing of the tangential components of the electric field. Hence, we have

$$
\begin{gathered}
\sum_{l=1}^{2} \sum_{q=1}^{2} \sum_{n^{\prime}=0}^{\infty} F_{m n^{\prime} q}^{(l)} \int_{0}^{\pi} A_{m n q}^{e} z_{n}^{(l)}\left(k_{q} a_{2}\right) P_{n^{\prime}}^{m}\left(\cos \theta_{k}\right) k_{q}^{2} \\
\times \sin \theta_{k} d \theta_{k}=0 ;
\end{gathered}
$$




$$
\begin{gathered}
\sum_{l=1}^{2} \sum_{q=1}^{2} \sum_{n^{\prime}=0}^{\infty} F_{m n^{\prime} q}^{(l)} \int_{0}^{\pi}\left[B_{m n q}^{e} R_{n}^{(l)}\left(k_{q} r\right)+C_{m n q}^{e} \frac{z_{n}^{(l)}\left(k_{q} r\right)}{r}\right]_{r=a_{2}} \\
P_{n^{\prime}}^{m}\left(\cos \theta_{k}\right) k_{q}^{2} \sin \theta_{k} d \theta_{k}=0 .
\end{gathered}
$$

In (8a) to (9b), $m$ and $n$ are two arbitrary integers; the radial function $R_{n}^{(l)}(x)$ has the following expression:

$$
R_{n}^{(l)}(x)=\frac{1}{x} \frac{d}{d x}\left[x z_{n}^{(l)}(x)\right] .
$$

From (8a) to (9b), it shows that

(i) firstly, there are six equations and six unknown coefficients, namely $F_{m n^{\prime} q}^{(l)}$ (where $q=1,2$ and $l=1$,2 ), $A_{m n}^{s}$, and $B_{m n}^{s}$; the unknown coefficients of electromagnetic fields in the plasma anisotropic spherical medium can be obtained;

(ii) then, the radar cross-section of an anisotropic plasma-coated conducting sphere by a plane wave can be derived.

\section{Numerical Results and Discussion}

In the last section, we have presented the necessary theoretical formulation of the electromagnetic fields of a plane wave scattered by an anisotropic plasma-coated conducting sphere. To gain more physical insight into the problem, we will provide, in this section, some numerical solutions to the problem of a plane electromagnetic wave scattered by an anisotropic plasma-coated conducting sphere.

To demonstrate the accuracy of the solutions achievable by using the present method, we compare bistatic radar cross-sections (RCSs) in E-plane (xoz-plane as shown in Figure 1) and $H$-plane ( $y o z$-plane as shown in Figure 1) to the FE-BI-MLFMA [11], as shown in Figure 2. The series in $(8 \mathrm{a}),(8 \mathrm{~b}),(8 \mathrm{c}),(8 \mathrm{~d}),(9 \mathrm{a})$, and (9b) converges rapidly, and it is sufficient to take $N=8$ as the upper limit of the summation indices $n$ and $n^{\prime}$. Certainly, similar as in [13-15], it should be pointed out that the convergence rate or the upper limit of the summation depends on the electrical dimension of the sphere (with respect to the wavelength). In Figure 2, the RCSs in both $E$ - and $H$-planes using the formulations in this paper are compared to FE-BI-MLFMA. A good agreement of the RCS results is achieved between those two methods, where the permittivity tensor elements of anisotropic plasma medium are characterized by $\epsilon_{1}=3 \epsilon_{0}, \epsilon_{2}=2 i \epsilon_{0}$, and $\epsilon_{3}=4 \epsilon_{0}$. And the electric size of plasma-coated conductor sphere is chosen as $k_{0} a_{1}=1.3 \pi$ and $k_{0} a_{2}=1.2 \pi$. It partially verifies that the proposed method and the Fortran code developed in this paper are correct.

After the validation studies, we obtain some new results unavailable elsewhere in literature. Three examples are considered herein, and their radar cross-sections are plotted in Figures 3-5.

Figure 3 depicts radar cross-sections of a more general anisotropic plasma-coated conducting sphere; the plasma is lossless and the permittivity tensor elements of the plasma

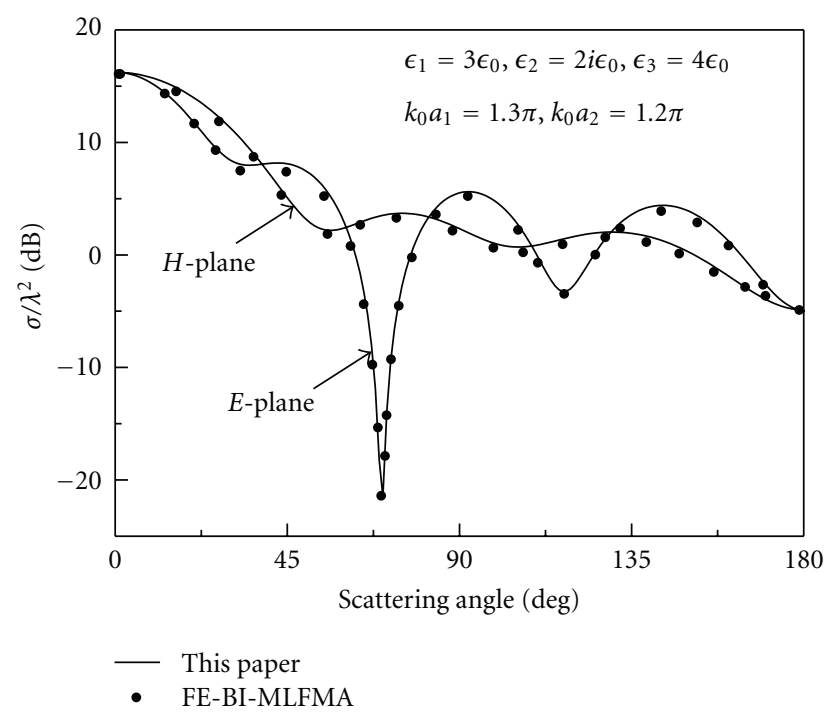

FIGURE 2: Radar cross-sections (RCSs) versus scattering angle $\theta$ (in degrees): results of this paper (solid curve) and FE-BI-MLFMA method. The electric dimensions of the outer and inner spherical surfaces are chosen as $k_{0} a_{1}=1.3 \pi$ and $k_{0} a_{2}=1.2 \pi$ while the permittivity tensor elements of the plasma are assumed to be $\epsilon_{1}=3 \epsilon_{0}$, $\epsilon_{2}=2 i \epsilon_{0}$, and $\epsilon_{3}=4 \epsilon_{0}$, respectively.

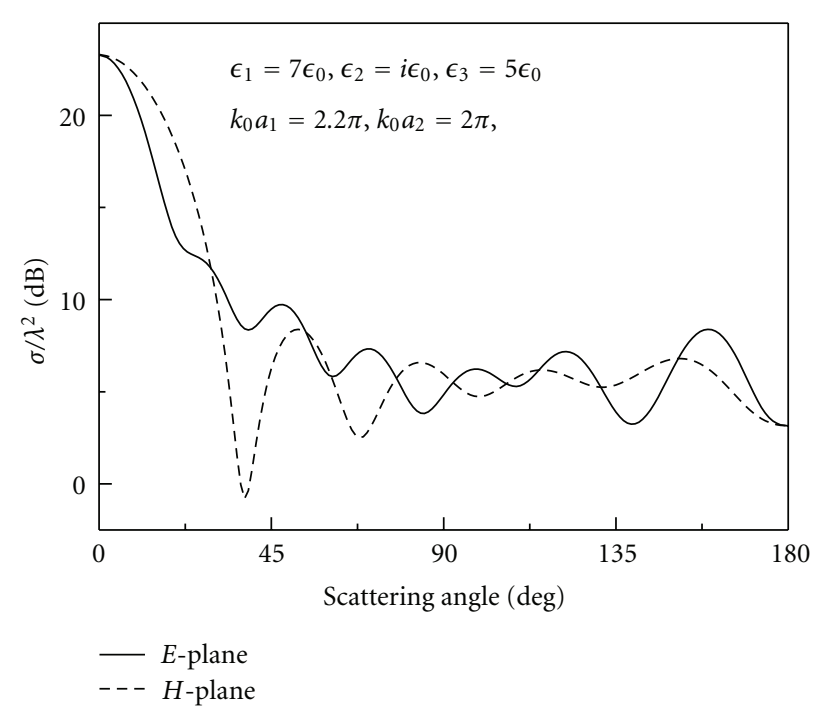

FIGURE 3: Radar cross-sections (RCSs) versus scattering angle $\theta$ (in degrees) in the $E$-plane (solid curve) and in the $H$-plane (dashed curve). The electric dimensions of the outer and inner spherical surfaces are chosen as $k_{0} a_{1}=2.2 \pi$ and $k_{0} a_{2}=2 \pi$ while the permittivity tensor elements of the plasma are assumed to be $\epsilon_{1}=7 \epsilon_{0}$, $\epsilon_{2}=i \epsilon_{0}$, and $\epsilon_{3}=5 \epsilon_{0}$, respectively.

are characterized by $\epsilon_{1}=7 \epsilon_{0}, \epsilon_{2}=i \epsilon_{0}$, and $\epsilon_{3}=5 \epsilon_{0}$; the electric size of the plasma anisotropic spherical shell is chosen as $k_{0} a_{1}=2.2 \pi$ and $k_{0} a_{2}=2 \pi$. Figure 4 depicts a thick plasma anisotropic medium coated conducting sphere, and the plasma is lossy, the permittivity tensor elements of the plasma are characterized by $\epsilon_{1}=(7+0.2 i) \epsilon_{0}, \epsilon_{2}=i \epsilon_{0}$, and $\epsilon_{3}=(5+0.1 i) \epsilon_{0}$, the electric size of the plasma anisotropic 


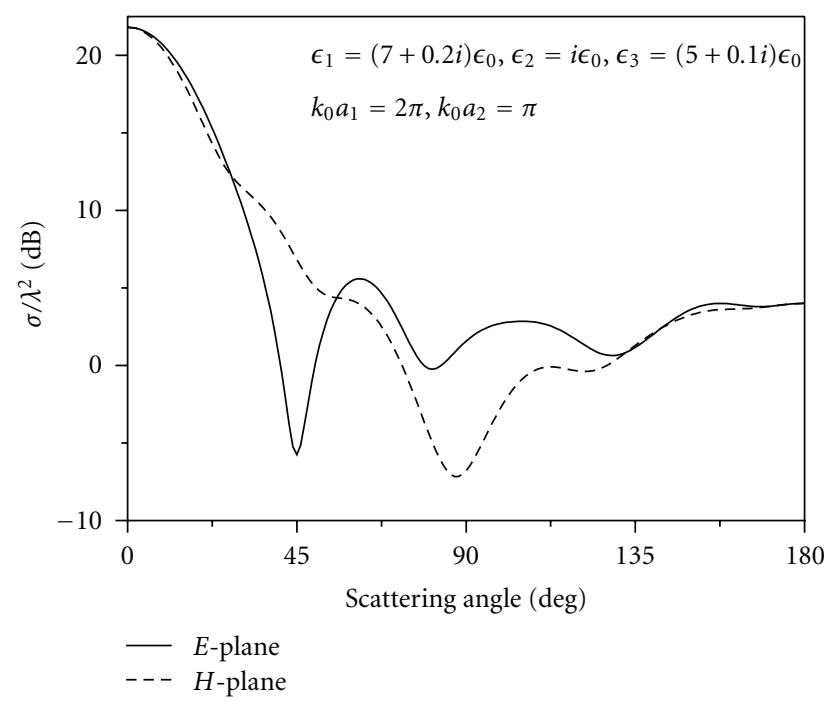

FIgURE 4: Radar cross-sections (RCSs) versus scattering angle $\theta$ (in degrees) in the $E$-plane (solid curve) and in the $H$-plane (dashed curve). The electric dimensions of the outer and inner spherical surfaces are chosen as $k_{0} a_{1}=2 \pi$ and $k_{0} a_{2}=\pi$ while the permittivity tensor elements of the plasma are assumed to be $\epsilon_{1}=(7+0.2 i) \epsilon_{0}$, $\epsilon_{2}=i \epsilon_{0}$, and $\epsilon_{3}=(5+0.1 i) \epsilon_{0}$, respectively.

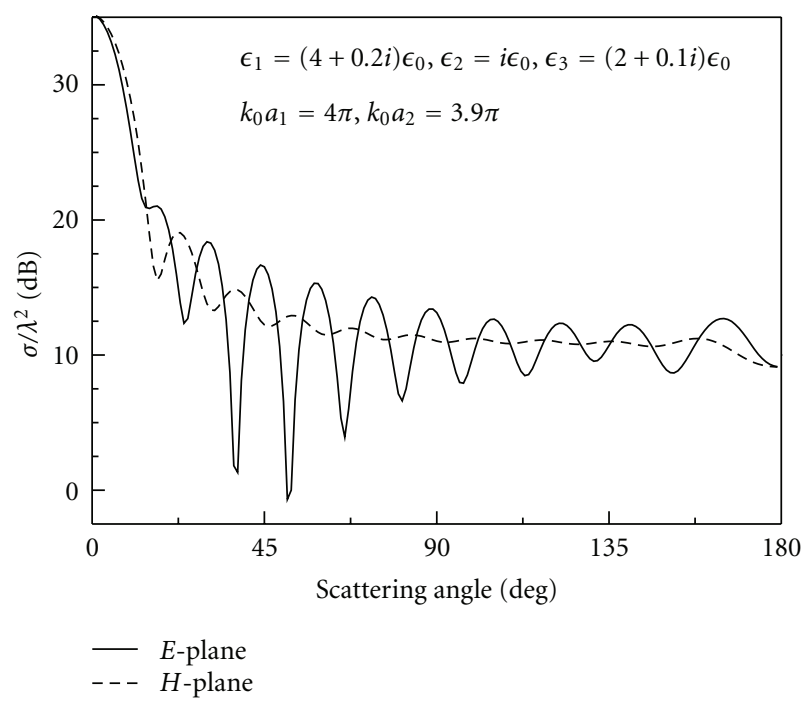

Figure 5: Radar cross-sections (RCSs) versus scattering angle $\theta$ (in degrees) in the $E$-plane (solid curve) and in the $H$-plane (dashed curve). The electric dimensions of the outer and inner spherical surfaces are chosen as $k_{0} a_{1}=4 \pi$ and $k_{0} a_{2}=3.2 \pi$ while the permittivity tensor elements of the plasma are assumed to be $\epsilon_{1}=$ $(4+0.2 i) \epsilon_{0}, \epsilon_{2}=i \epsilon_{0}$, and $\epsilon_{3}=(2+0.1 i) \epsilon_{0}$, respectively.

spherical shell is chosen as $k_{0} a_{1}=2 \pi$ and $k_{0} a_{2}=\pi$. In those two figures, the maximum number $n^{\prime}$ in $(8 \mathrm{a}),(8 \mathrm{~b}),(8 \mathrm{c})$, $(8 d),(9 a)$, and $(9 b)$ to achieve a good convergence is found to be 12 (slightly larger than the quantity of Figure 2 due to the increased electric dimensions).

To illustrate further applicability of the solution to electromagnetic scattering by an electrically large-sized aniso- tropic plasma-coated conducting sphere (e.g., in its resonance region), the radar cross-sections of a coated sphere of relatively large electric size with $k_{0} a_{1}=4 \pi$ and $k_{0} a_{2}=$ $3.9 \pi$, under the illumination by an incident plane wave, are obtained and depicted in both the $E$-plane and the $H$-plane in Figure 5. The permittivity tensor parameters used for this case are $\epsilon_{1}=(4+0.2 i) \epsilon_{0}, \epsilon_{2}=i \epsilon_{0}$, and $\epsilon_{3}=(2$ $+0.1 i) \epsilon_{0}$. As the electric dimension of our radius of the anisotropic plasma-coated conducting sphere is increased, the maximum number of $n^{\prime}$ used in $(8 \mathrm{a}),(8 \mathrm{~b}),(8 \mathrm{c}),(8 \mathrm{~d}),(9 \mathrm{a})$, and $(9 \mathrm{~b})$ must be significantly increased to 16 to achieve the convergence.

From the above discussions, it is seen and concluded that

(i) the radar cross-sections (RCS's) $\left(\sigma / \lambda^{2}\right)$ in both the $E$ and $H$-planes vary with the scattering angles;

(ii) for a medium-sized anisotropic plasma-coated conducting sphere, the RCS's in both the $E$ - and $H$-planes vary oscillatingly with amplitude decreasing with the scattering angle;

(iii) as the large-size for out radius of an anisotropic plasma-coated conducting sphere, the RCS's in the E- and $H$-planes have almost the same main beam width.

\section{Conclusions}

The spherical vector wave functions expansion technique is successfully applied in the present work for an analytical solution to the problem of plane wave scattering by an anisotropic plasma-coated conducting sphere in this paper. The solution has only one-dimensional integral which can be easily evaluated. The theoretical analysis shows that when the radius of conducting sphere approaches zero, the results of present formulation can be reduced to those of a single plasma anisotropic sphere. In addition, the general numerical results, including the lossy plasma-coated conducting sphere and resonance region, are given.

\section{Acknowledgments}

The author is greatly indebted to Professor Sheng X. Q. and Dr. Peng Z. in Beijing Institute of Technology for sending us their data. This work is partially supported by Grant no. 60971047 of National Natural Science Foundation of China (NSFC), and Grant no. Y1080730 of Natural Science Foundation of Zhejiang Province of China.

\section{References}

[1] J. Rose David and C. Melvile, Plasmas and Controlled Fusion, John Wiley \& Sons, New York, NY, USA, 1961.

[2] J. R. Wait, "Some boundary value problems involving plasma media," Journal of Research of the National Bureau of Standards, vol. 65 , pp. 137-150, 1961.

[3] K. C. Yeh and C. H. Liu, Theory of Ionosphere Waves, Academic Press, New York, NY, USA, 1972.

[4] H. C. Chen and D. K. Cheng, "Scattering of electromagnetic waves by an anisotropic plasma-coated conducting cylinder," 
IEEE Transactions on Antennas and Propagation, vol. 12, no. 3, pp. 348-353, 1964.

[5] R. D. Graglia and P. L. E. Uslenghi, "Electromagnetic scattering from anisotropic material Part I: general theory," IEEE Transactions on Antennas and Propagation, vol. 32, no. 8, pp. 867869, 1984.

[6] A. Taflove, "Review of the formulation and applications of the finite-difference time-domain method for numerical modeling of electromagnetic wave interactions with arbitrary structures," Wave Motion, vol. 10, no. 6, pp. 547-582, 1988.

[7] X. B. Wu and K. Yasumoto, "Three-dimensional scattering by an infinite homogeneous anisotropic circular cylinder: an analytical solution," Journal of Applied Physics, vol. 82, no. 5, pp. 1996-2003, 1997.

[8] R. D. Graglia, P. L. E. Uslenghi, and R. S. Zich, "Moment method with isoparametric elements for three-dimensional anisotropic scatterers," Proceedings of the IEEE, vol. 77, no. 5, pp. 750-760, 1989.

[9] H. F. Ma, J. F. Zhang, X. Chen, Q. Cheng, and T. J. Cui, "CGFFT algorithm for three-dimensional inhomogeneous and biaxial metamaterials," Waves in Random and Complex Media, vol. 19, no. 1, pp. 49-64, 2009.

[10] V. V. Varadan, A. Lakhtakia, and V. K. Varadan, "Scattering by three-dimensional anisotropic scatterers," IEEE Transactions on Antennas and Propagation, vol. 37, no. 6, pp. 800-802, 1989.

[11] X. Q. Sheng and Z. Peng, "Analysis of scattering by large objects with off-diagonally anisotropic material using finite element-boundary integral-multilevel fast multipole algorithm," IET Microwaves, Antennas and Propagation, vol. 4, pp. 492500, 2010.

[12] S. N. Papadakis, N. K. Uzunoglu, and C. N. Capsalis, "Scattering of a plane wave by a general anisotropic dielectric ellipsoid," Journal of the Optical Society of America A, vol. 7, pp. 991-997, 1990.

[13] Y. L. Geng, X. B. Wu, and L. W. Li, "Analysis of electromagnetic scattering by a plasma anisotropic sphere," Radio Science, vol. 38, no. 6, Article ID 1104, 12 pages, 2003.

[14] Y. L. Geng, X. B. Wu, L. W. Li, and B. R. Guan, "Electromagnetic scattering by an imhomogeneous plasma anisotropic sphere of multilayers," IEEE Transactions on Antennas and Propagation, vol. 53, no. 12, pp. 3982-3989, 2005.

[15] Y. L. Geng, X. B. Wu, L. W. Li, and B. R. Guan, "Mie scattering by a uniaxial anisotropic sphere," Physical Review E, vol. 70, no. 5, Article ID 056609, 8 pages, 2004.

[16] D. Sarkar and N. J. Halas, "General vector basis function solution of Maxwell's equations," Phyical Rewiew E, vol. 56, pp. 1102-1997, 1997. 

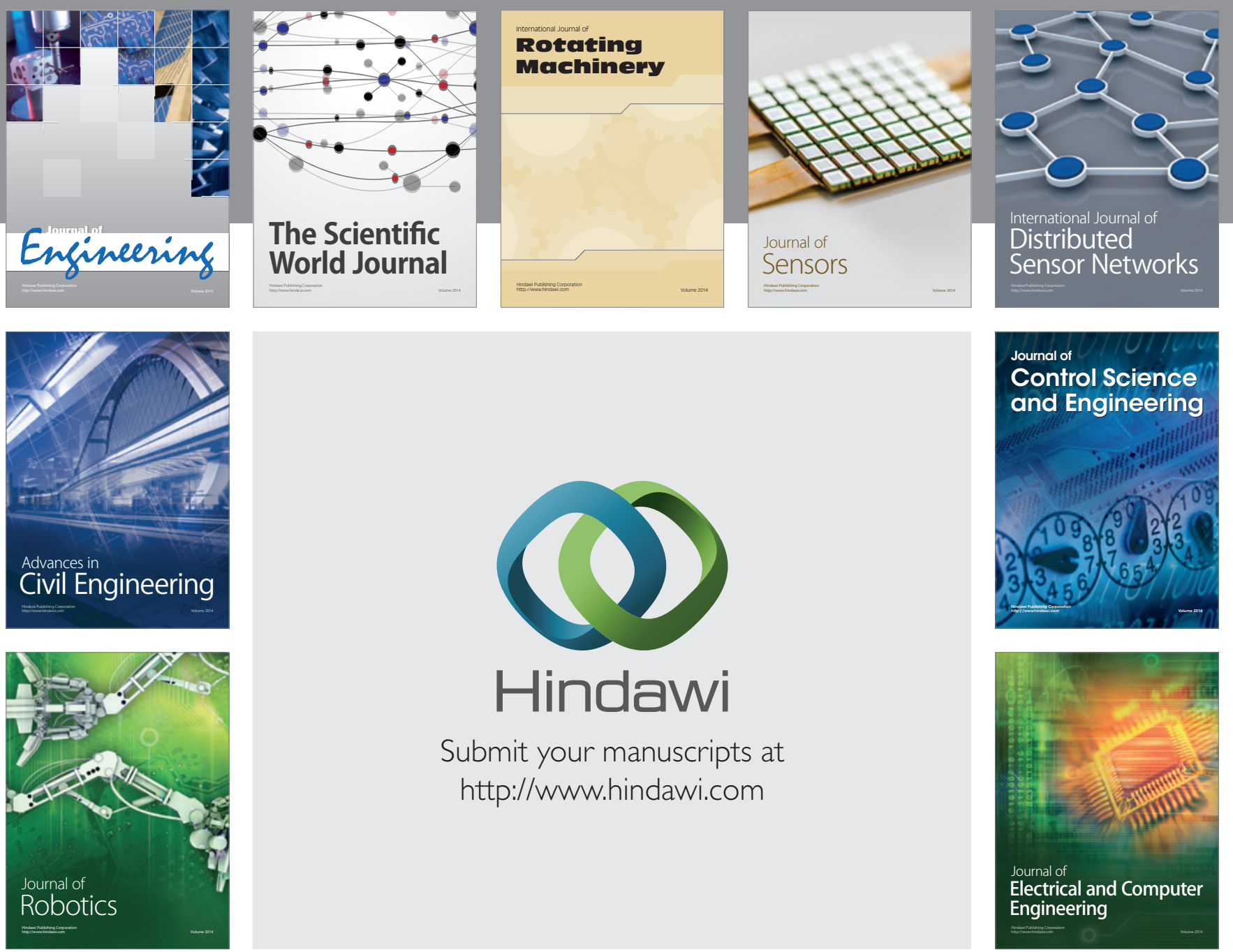

Submit your manuscripts at

http://www.hindawi.com
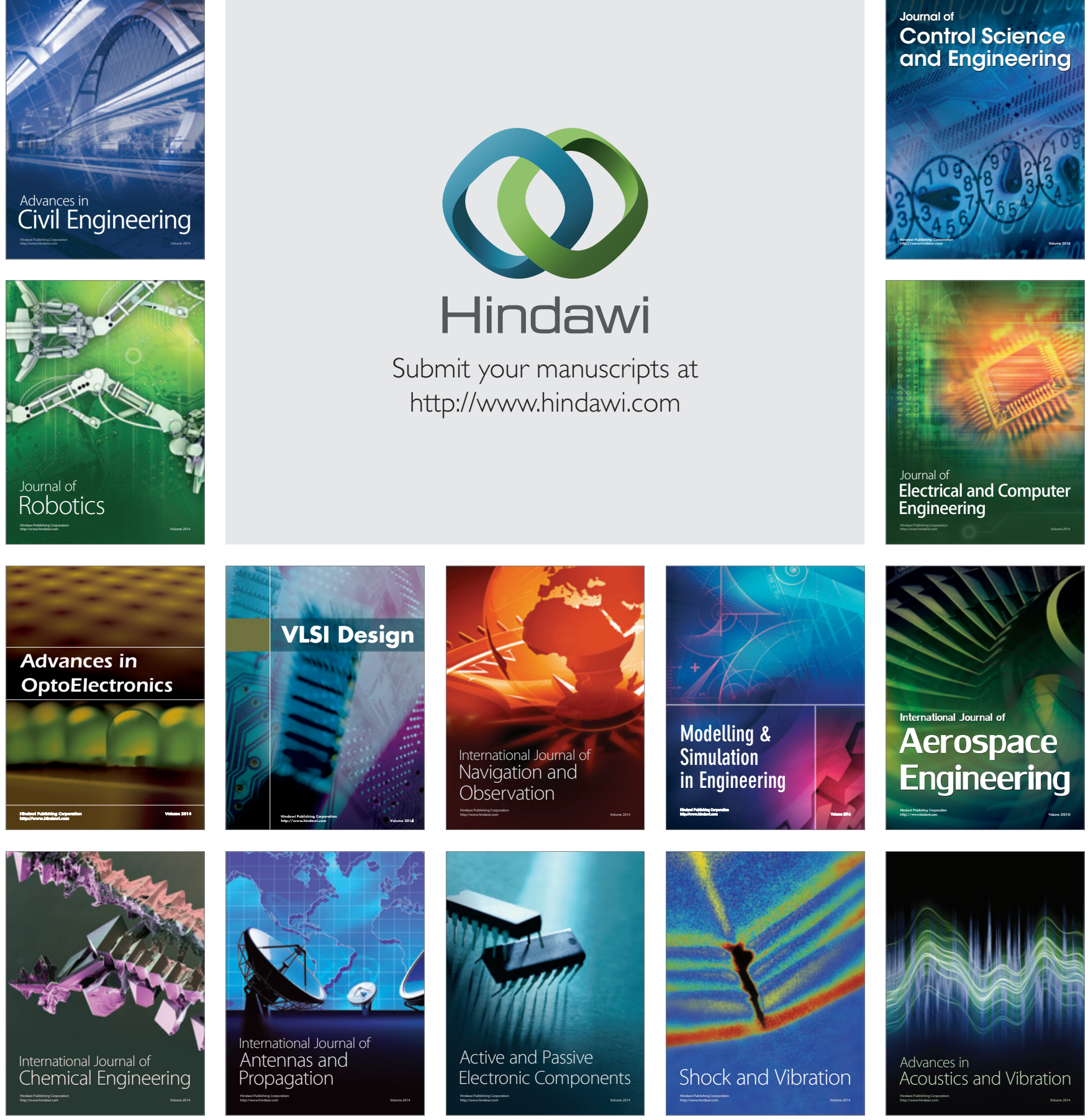